\title{
Relativistic current collection by a cylindrical Langmuir probe
}

\author{
G. Sánchez-Arriaga and J. R. Sanmartín \\ Departamento de Física Aplicada, Escuela Técnica Superior de Ingenieros Aeronáuticos, \\ Universidad Politécnica de Madrid, Plaza de Cardenal Cisneros 3, 28040 Madrid, Spain
}

(Received 27 March 2012; accepted 10 May 2012; published online 19 June 2012)

The current $I$ to a cylindrical Langmuir probe with a bias $\Phi_{p}$ satisfying $\beta \equiv e \Phi_{p} / m_{e} c^{2} \sim O(1)$ is discussed. The probe is considered at rest in an unmagnetized plasma composed of electrons and ions with temperatures $k T_{e} \sim k T_{i} \ll m_{e} c^{2}$. For small enough radius, the probe collects the relativistic orbital-motion-limited (OML) current $I_{O M L}$, which is shown to be larger than the nonrelativistic result; the OML current is proportional to $\beta^{1 / 2}$ and $\beta^{3 / 2}$ in the limits $\beta \ll 1$ and $\beta \gg 1$, respectively. Unlike the non-relativistic case, the electron density can exceed the unperturbed density value. An asymptotic theory allowed to compute the maximum radius of the probe to collect OML current, the sheath radius for probe radius well below maximum and how the ratio $I / I_{O M L}$ drops below unity when the maximum radius is exceeded. A numerical algorithm that solves the Vlasov-Poisson system was implemented and density and potential profiles presented. The results and their implications in a possible mission to Jupiter with electrodynamic bare tethers are discussed. (C) 2012 American Institute of Physics. [http://dx.doi.org/10.1063/1.4729662]

\section{INTRODUCTION}

Langmuir probes ${ }^{1}$ have been used for decades to make plasma diagnostics in laboratory and space conditions. The method rests on theoretical models, which provides the link between certain plasma parameters, such as density and temperature, and the measured current-voltage characteristics. Probe modeling also applies to space electrodynamic bare tethers, which freely collect charge from the ambient plasma and act like a giant Langmuir probe (lengths of the order of kilometers) under bias arising from the motional field $\mathbf{v}_{\text {rel }} \times$ $\mathbf{B}$ induced by the relative tether-plasma motion $\left(\mathbf{v}_{\text {rel }}\right.$ is the relative velocity and $\mathbf{B}$ is the ambient magnetic field). Langmuir probe analysis received great attention in the past and the current collection and sheath structure have been determined for both monoenergetic ${ }^{2,3}$ and Maxwellian ${ }^{4-6}$ distribution functions for the attracted species. Different effects, involving the ambient magnetic field ${ }^{7}$ and the self-field in the tether case ${ }^{8,9}$ or the relative velocity between the probe and the plasma ${ }^{10,11}$ have been also studied.

Relativistic effects, which become important when the probe potential $\Phi_{p}$ is high enough to have $e \Phi_{p} \sim m_{e} c^{2}$, are typically negligible for Langmuir probes operating in laboratory plasmas and also for bare tethers flying around the Earth. However, the situation is different for a recently proposed mission to Jupiter $^{12}$; a bare tape-tether would attain a circular orbit below the Jovian Radiation Belts and the Halo ring by using the Lorentz drag on the passively induced current to first brake the spacecraft into a near-parabolic orbit with perifocus around $1.4 R_{J}$ and then progressively lower the apojove through a series of drag arcs around the perijove passes. Such a scheme, as opposite to previous missions to Jupiter like Pioneer 10 and 11, Voyager 1 and 2, Ulysses, Cassini, and New Horizons, would allow to slowly descend in equatorial orbit through the inner magnetosphere of Jupiter over a period of months and provide a wealth of knowledge about Giant planets.
A tether with length $L=50 \mathrm{~km}$ and flying in a near parabolic, prograde orbit with perijove $r_{p}=1.4 R_{J}$ after capture would find a typical magnetic field value $B \approx 4.2$ $\times\left(R_{J} / r_{p}\right)^{3} \approx 1.5 \times 10^{-4} \mathrm{~T}$ and tether-to-plasma relative velocity $v_{\text {rel }}=\sqrt{2 \mu_{J} / r_{p}}-\Omega_{J} r_{p} \approx 33 \mathrm{~km} / \mathrm{s}$. The tether potential, slightly reduced by ohmic and $\mathbf{B}$ tilt-related angle effects, would be around $\Phi_{p}=v_{r e l} B L \lesssim 0.25 \mathrm{MV}$; here $\mu_{J}$ and $\Omega_{J}$ are the Jupiter gravitational parameter and spin velocity, respectively. The ratio $\beta \equiv e \Phi_{p} / m_{e} c^{2}$ would be near 0.5 , thus, making for sensible relativistic effects. Independently of corrections to the collected current, which are discussed in this work, the penetration length of energetic electrons into materials raises an issue for a tether mission at Jupiter. For $0.2 \mathrm{MeV}$ electrons the penetration length in aluminum can be as high as $0.25 \mathrm{~mm}$ (Fig. 6.4 in Ref. 13), thus suggesting to reduce the length of the tether and increase its width. This constraint must then be considered together with the originally discussed tether bowing and tensile stress, heating and radiation dose. ${ }^{14}$

Since electrons would then reach the anodic tip with moderately relativistic velocities, it is required to extend the orbital-motion-limited (OML) regime of cylindrical Langmuir probes to a relativistic subregime. In Sec. II we consider those relativistic effects that can be determined from simple OML-regime basics, in particular a modified OMLcurrent law itself. In Sec. III we use the asymptotic theory presented in Refs. 5 and 6 to compute the maximum probe radius-to-Debye length ratio for the OML regime to hold, the sheath radius for thin probes and the current collected when the maximum radius is exceeded. In Sec. IV we numerically derive potential and electron density profiles using a coupled Vlasov-Poisson solver. Conclusions are discussed in Sec. V.

\section{THE RELATIVISTIC OML REGIME}

Although a tape would be more efficient in a possible mission, we here consider for simplicity a cylinder of radius 
$R$ at bias $\Phi_{p}$ immersed in a collisionless, unmagnetized, Maxwellian plasma of unperturbed density $N_{0}$. The cylinder is sufficiently long to ignore edge effects and the plasma is composed of electrons and ions with temperatures $T_{e}$ and $T_{i}$, respectively. In the situation of interest here, $e \Phi_{p} \sim m_{e} c^{2}$ $\gg k T_{i} \sim k T_{e}$, the determination of the current collection involves the consistent solution of (i) the Poisson equation in cylindrical coordinates ( $\mathrm{z}$ is along the probe axis),

$$
\frac{\lambda_{D i}^{2}}{r} \frac{d}{d r}\left(r \frac{d}{d r} \frac{e \Phi}{k T_{i}}\right)=\frac{N_{e}}{N_{0}}-\frac{N_{i}}{N_{0}} \approx \frac{N_{e}}{N_{0}}-\exp \left(-\frac{e \Phi}{k T_{i}}\right),
$$

with boundary conditions $\Phi=\Phi_{p}$ at $r=R$ and $\Phi \rightarrow 0$ as $r \rightarrow \infty$ and (ii) the stationary relativistic Vlasov equation for the electron distribution function $f(\mathbf{r}, \mathbf{v})$

$$
v_{r} \frac{\partial f}{\partial r}+\left(\frac{v_{\theta} p_{\theta}}{r}+e \frac{\partial \Phi}{\partial r}\right) \frac{\partial f}{\partial p_{r}}-\frac{v_{\theta} p_{r}}{r} \frac{\partial f}{\partial p_{\theta}}=0,
$$

with $f(\mathbf{r}, \mathbf{v}) \rightarrow f_{M}\left(v_{\infty}\right)$ (undisturbed Maxwellian) as $r \rightarrow \infty$. In Eq. (1), the Boltzmann law holds for the ion density $N_{i}$ at the case of interest $e \Phi_{p} \gg k T_{i}$ whereas the electron density $N_{e}$ requires integrating $f(\mathbf{r}, \mathbf{v})$ in velocity space. Here $\lambda_{D i}$ is the ion Debye length. Velocity and momentum are related by $\mathbf{p}=m_{e} \gamma \mathbf{v}$ where $\gamma$ is given by

$$
\begin{aligned}
\gamma & =\sqrt{1+\frac{|\mathbf{p}|^{2}}{m_{e}^{2} c^{2}}}=\sqrt{1+\frac{\left|\mathbf{p}_{\perp}\right|^{2}}{m_{e}^{2} c^{2}}} \sqrt{1+\frac{p_{z}^{2}}{m_{e}^{2} c^{2}+\left|\mathbf{p}_{\perp}\right|^{2}}} \\
& \equiv \gamma_{\perp} \sqrt{1+\frac{p_{z}^{2}}{m_{e}^{2} c^{2}+\left|\mathbf{p}_{\perp}\right|^{2}}}
\end{aligned}
$$

and $\mathbf{p}_{\perp}$ is the momentum transverse to the z-axis.

As in Ref. 5, the Vlasov characteristic equations show that the distribution function $f(\mathbf{r}, \mathbf{v})$, the energy $E=m_{e} c^{2}(\gamma-1)-e \Phi$, the angular momentum $J=r p_{\theta}$, and $p_{z}$ are all conserved along the orbits. Therefore, ignoring possible trapped particles, we can set $f(\mathbf{r}, \mathbf{v})=f_{M}\left(v_{\infty}\right)$ if the $\mathbf{r}, \mathbf{v}$ orbit traced back in time reaches infinity and $f(\mathbf{r}, \mathbf{v})=0$ otherwise. This property extremely simplifies the calculation since it allows to write the electron density as

$$
\begin{aligned}
\frac{N_{e}}{N_{0}} & =\int \frac{d \mathbf{p}}{\left(2 \pi k T_{e} m_{e}\right)^{3 / 2}} \times \exp \left(\frac{-E}{k T_{e}}\right) \\
& =\int \frac{d \mathbf{p}_{\perp}}{\left(2 \pi k T_{e} m_{e}\right)^{3 / 2}} \times \exp \left(\frac{m_{e} c^{2}+e \Phi}{k T_{e}}\right) \times Q,
\end{aligned}
$$

where

$$
Q \equiv \int_{-\infty}^{+\infty} \exp \left(-\frac{m_{e} c^{2} \gamma_{\perp}}{k T_{e}} \sqrt{1+\frac{p_{z}^{2}}{m_{e}^{2} c^{2}+\left|\mathbf{p}_{\perp}\right|^{2}}}\right) d p_{z}
$$

Since $m_{e} c^{2} \gg k T_{e}$, the square root inside the exponential in Eq. (5) can be expanded in a Taylor series to yield

$$
Q \approx \sqrt{2 \pi k T_{e} m_{e} \gamma_{\perp}} \exp \left(-\frac{m_{e} c^{2} \gamma_{\perp}}{k T_{e}}\right)
$$

Using Eq. (6) and defining $E_{\perp} \equiv m_{e} c^{2}\left(\gamma_{\perp}-1\right)-e \Phi$, which is also conserved, make Eq. (4) read

$$
\frac{N_{e}}{N_{0}}=\int \frac{\sqrt{\gamma_{\perp}} \exp \left(-E_{\perp} / k T_{e}\right) d \mathbf{p}_{\perp}}{2 \pi k T_{e} m_{e}}
$$

and with the change of variables $\left(p_{r}, p_{\theta}\right) \rightarrow\left(E_{\perp}, J\right)$ become

$$
\frac{N_{e}}{N_{0}} \approx\left(1+\frac{e \Phi}{m_{e} c^{2}}\right)^{3 / 2} \iint \frac{d E_{\perp}}{2 \pi k T_{e}} \frac{d J \exp \left(-E_{\perp} / k T_{e}\right)}{\sqrt{J_{r}^{2}\left(E_{\perp}\right)-J^{2}}} .
$$

In Eq. (8), we took into account the limit of interest $m_{e} c^{2} \gg$ $k T_{e}$ to ignore the term $E_{\perp} / m_{e} c^{2}$ in the parenthesis that appears outside the integral. As compared with the nonrelativistic calculation, ${ }^{5}$ the electron density is modified by the factor outside the integral and by the $J_{r}\left(E_{\perp}\right)$ definition

$$
J_{r}^{2}\left(E_{\perp}\right) \equiv m_{e}^{2} c^{2} r^{2}\left[\left(1+\frac{e \Phi+E_{\perp}}{m_{e} c^{2}}\right)^{2}-1\right] .
$$

Following Ref. 5, where a detailed discussion about integral limits and possible orbits can be found, we carry out the J-integral to find

$$
\begin{aligned}
\frac{N_{e}(r)}{N_{0}}= & \left(1+\frac{e \Phi}{m_{e} c^{2}}\right)^{3 / 2} \int_{0}^{+\infty} \frac{d E_{\perp}}{\pi k T_{e}} \exp \left(-\frac{E_{\perp}}{k T_{e}}\right) \\
& \times\left[2 \arcsin \left(\frac{J_{r}^{*}\left(E_{\perp}\right)}{J_{r}\left(E_{\perp}\right)}\right)-\arcsin \left(\frac{J_{R}^{*}\left(E_{\perp}\right)}{J_{r}\left(E_{\perp}\right)}\right)\right],
\end{aligned}
$$

where the function

$$
J_{r}^{*}\left(E_{\perp}\right) \equiv \operatorname{minimum}\left[J_{r^{\prime}}\left(E_{\perp}\right) ; r^{\prime} \geq r\right]
$$

is introduced to exclude electrons with an angular momentum too large to reach the position $\mathrm{r}$. We remark that the definitions of $E_{\perp}, J$, and $J_{r}$ give $r^{2} p_{r}^{2}=J_{r}^{2}-J^{2}$. Therefore, electrons in the range $J_{r}^{*}\left(E_{\perp}\right)<J<J_{r}\left(E_{\perp}\right)$ would have $p_{r}^{2}$ negative at some $r^{\prime}$ in the range $r<r^{\prime}<\infty$ and do not contribute to the density at the position $r$. This is called an effective potential barrier at $r$ for energy $E_{\perp}$.

In the absence of potential barrier and sink (probe radius satisfying $\left.R / \lambda_{D} \rightarrow 0\right)$, Eq. (10) gives $N_{e} / N_{0}=(1+e \Phi /$ $\left.m_{e} c^{2}\right)^{3 / 2}$, which can be considered the relativistic extension of a well-known and simple result found in Ref. 15; unlike the classical result in Ref. 15, relativistic effects allow to have an electron density over $N_{0}$ for two-dimensional potential wells and isotropic distribution functions at infinity.

Again, with a $p_{z}$ integration and the change $p_{r}, p_{\theta} \rightarrow E_{\perp}, J$, one finds the current collected by the probe

$$
\begin{aligned}
I & =2 \pi R L e \int v_{r} f d \mathbf{p} \\
& =2 R L e c N_{0} \sqrt{1+\beta} \int_{0}^{\infty} \exp \left(-\frac{E_{\perp}}{k T_{e}}\right) \frac{J_{R}^{*}}{R m_{e} c} \frac{d E_{\perp}}{k T_{e}},
\end{aligned}
$$

with $\beta \equiv e \Phi_{p} / m_{e} c^{2}$. Since $J_{R}^{*}\left(E_{\perp}\right) \leq J_{R}\left(E_{\perp}\right)$, current is maximum under the condition $J_{R}^{*}\left(E_{\perp}\right)=J_{R}\left(E_{\perp}\right)$, for $0 \leq E_{\perp}<\infty$; this is the OML regime corresponding to no 
potential barriers for radius $R$. We note that $J_{R}^{*}\left(E_{\perp}\right)=J_{R}\left(E_{\perp}\right)$ in the entire range $0 \leq E_{\perp}<\infty$ is fulfilled if $J_{R}^{*}(0)=J_{R}(0)$. Taking into account Eqs. (9) and (11), the condition $J_{R}^{*}(0)$ $=J_{R}(0)$ requires the potential to satisfy

$$
\frac{\Phi}{\Phi_{p}} \geq \frac{-1+\sqrt{1+\left[(1+\beta)^{2}-1\right](R / r)^{2}}}{\beta}
$$

and far away from the probe $r / R \gg 1$

$$
\frac{\Phi}{\Phi_{p}} \geq \frac{(1+\beta)^{2}-1}{2 \beta}\left(\frac{R}{r}\right)^{2}>\left(\frac{R}{r}\right)^{2}
$$

which recovers the non-relativistic condition $\Phi / \Phi_{p} \geq R^{2} / r^{2}$ at low $\beta .^{5}$ The OML current is obtained using $J_{R}^{*}\left(E_{\perp}\right)$ $=J_{R}\left(E_{\perp}\right) \approx J_{R}(0)$ in Eq. (12) to find

$$
I_{O M L}=2 R \operatorname{Rec} N_{0} \sqrt{1+\beta} \sqrt{(1+\beta)^{2}-1} .
$$

In the non-relativistic limit $\beta \ll 1$, Eq. (15) becomes the well known formula $I=2 R L e N_{0} \sqrt{2 e \Phi_{p} / m_{e}}(I \sim \sqrt{\beta})$ whereas for $\beta \gg 1$ we have $I=2 R L e N_{0} \sqrt{2 e \Phi_{p} / m_{e}} \times e \Phi_{p} /$ $\sqrt{2} m_{e} c^{2}\left(I \sim \beta^{3 / 2}\right)$ [see panel (a) in Fig. 1]. From Eq. (10), we find the OML electron density at the probe,

$$
\left.\frac{N_{e}(r=R)}{N_{0}}\right|_{\text {OML }}=\frac{1}{2}(1+\beta)^{3 / 2} .
$$

Equation (16) is the relativistic extension to the formula given in Ref. 15, where very general results are presented for arbitrary convex cross section probes with isotropic distribution functions at infinity. The relativistic effects increase both the OML current and the electron density at the probe [see panels (a) and (b) in Fig. 1].

\section{ASYMPTOTIC ANALYSIS}

This section presents an extension to relativistic conditions of the asymptotic analysis carried out in Refs. 5 and 6. The different domains that appear in the analysis can be seen in Fig. 2
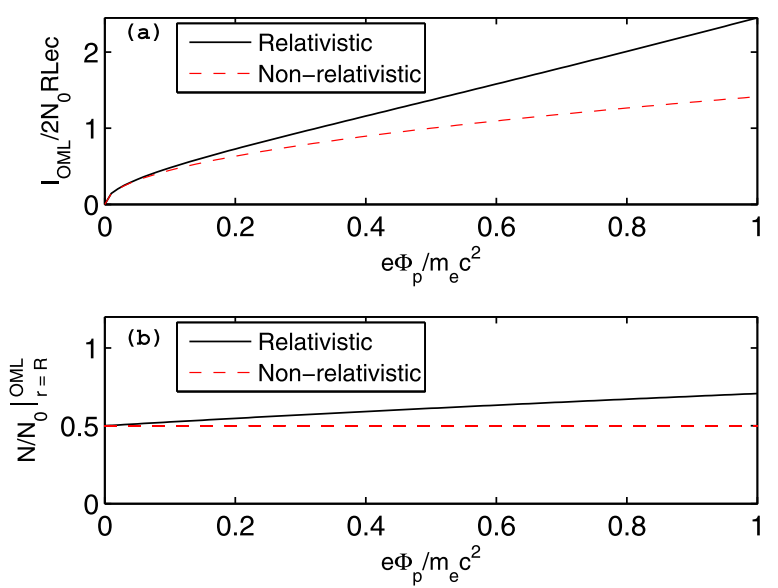

FIG. 1. Comparison of relativistic and non-relativistic normalized collected current [panel (a)] and normalized electron density at the probe [panel (b)] versus $e \Phi_{p} / m_{e} c^{2}$. (a)
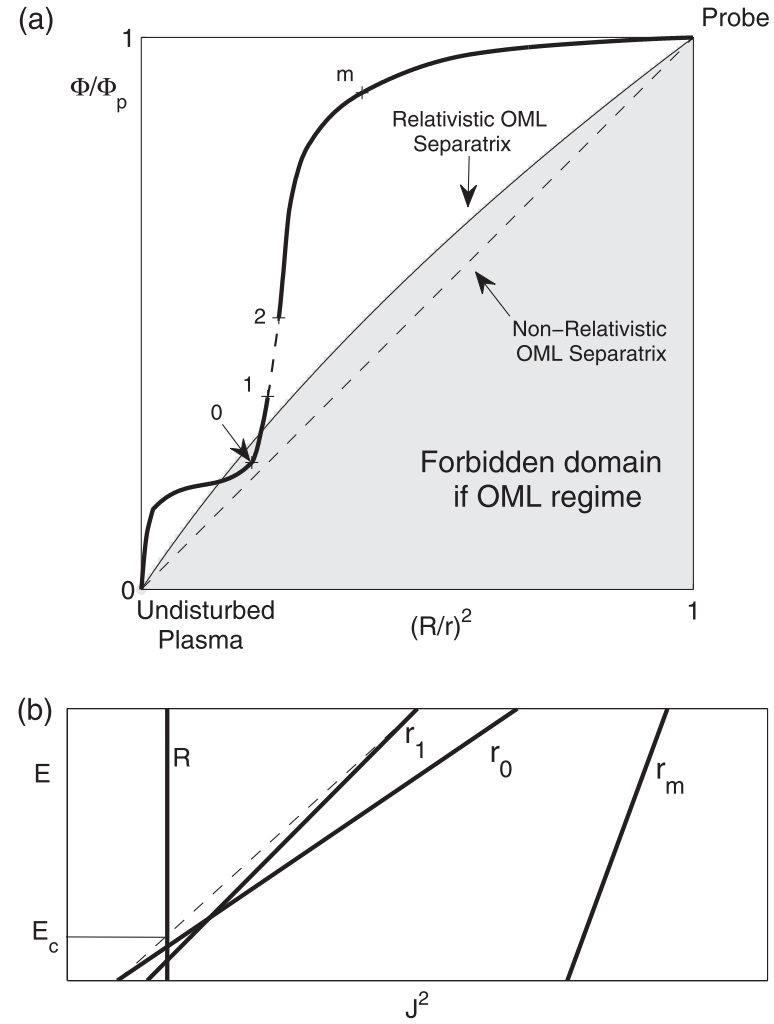

FIG. 2. Panel (a) shows $\Phi / \Phi_{p}$ versus $R^{2} / r^{2}$ for a probe with a radius larger than the maximum radius for the OML regime. The relativistic separatrix [Eq. (13)] is also shown. The plasma is quasineutral below point 1 and there is no potential barriers below point 0 . The figure is not drawn to scale (points 0,1 , and 2 would occur near the origin). Panel (b) displays the r-lines (solid lines) $J_{r}^{2}=J^{2}\left(E_{\perp}\right)$ at the probe radius and at points 0,1 , and $m$ indicated in panel (a). The envelope (dashed line) of the r-lines in the range $r_{1}<r<r_{0}$ and the energy $E_{c}$ where the envelope cuts the $R$-line are also shown.

[panel (a)] that shows $\Phi / \Phi_{p}$ versus $(R / r)^{2}$. Decreasing the radius from infinity to the probe we find the following domains: (i) $r>r_{0}$ quasineutral plasma without potential barriers, (ii) $r_{0}>r>r_{1}$ quasineutral plasma with potential barriers, (iii) transitional layers at $r_{1}$ and $r_{2}$, and (iv) $r_{2}>r>R$ corresponding to the sheath. Domains (i) and (ii) make up the presheath. The asymptotic method solves Eqs. (1) and (10) in the different domains by retaining only the dominant terms in each case and taking into account the appearance of potential barriers.

\section{A. Basic formulation}

\section{Quasineutral presheath}

Far away from the probe, one has $e \Phi(r) \ll m_{e} c^{2}$ and the relativistic effects play no role. Equations (9) and (10) become

$$
\begin{gathered}
J_{r}^{2}\left(E_{\perp}\right) \approx 2 m_{e} r^{2}\left(E_{\perp}+e \Phi\right), \\
\frac{N_{e}}{N_{0}} \approx \int_{0}^{\infty} \frac{d E_{\perp}}{\pi k T_{e}} \exp \left(-\frac{E_{\perp}}{k T_{e}}\right) \\
\times\left[2 \arcsin \left(\frac{J_{r}^{*}\left(E_{\perp}\right)}{J_{r}\left(E_{\perp}\right)}\right)-\arcsin \left(\frac{J_{R}^{*}\left(E_{\perp}\right)}{J_{r}\left(E_{\perp}\right)}\right)\right] .
\end{gathered}
$$

The condition of no potential barrier at $r$ now reads $r^{2} \Phi(r)<r^{\prime 2} \Phi\left(r^{\prime}\right)$ for $r<r^{\prime}<\infty$. $^{5}$ Since the results from the non-relativistic analysis can then be directly applied in 
this region, we will just give the essential equations required to find the collected current without a thorough justification. The details of the method and a discussion can be found in Refs. 5 and 6. As shown in Fig. 2, decreasing the radius from $\infty$, the following layers appear.

a. Quasineutral region without potential barriers. Faraway from the probe the plasma is quasineutral; making $N_{e} \approx N_{i}$ one finds $\Phi \sim 1 / r$ and there are no potential barriers. There exists a radius $r_{0}$ with potential value $\Phi_{0}$ where a potential barrier first appears [see panel (a) in Fig. 2]. We then can write $J_{r}^{*}\left(E_{\perp}\right)=J_{r}\left(E_{\perp}\right)$ in Eq. (18) and find the potential $\Phi_{0}$ in terms of $r_{0}$ by using $N_{e} \approx N_{i}$, reading

$$
\begin{aligned}
\exp \left(-\frac{e \Phi_{0}}{k T_{i}}\right)= & 1-\int_{0}^{\infty} \frac{d E_{\perp}}{\pi k T_{e}} \times \exp \left(-\frac{E_{\perp}}{k T_{e}}\right) \\
& \times \arcsin \left(\frac{J_{R}^{*}\left(E_{\perp}\right)}{J_{r_{0}}\left(E_{\perp}\right)}\right) .
\end{aligned}
$$

The determination of $J_{R}^{*}\left(E_{\perp}\right)$ in Eq. (19) requires a detail examination of the family of r-lines in the $E-J^{2}$ diagram [see panel (b) in Fig. 2]. In Ref. 6, it was demonstrated that $J_{R}^{*}\left(E_{\perp}\right)$ takes the form

$$
\begin{gathered}
J_{R}^{*}\left(E_{\perp}\right)=J_{\text {env }}\left(E_{\perp}\right) \quad \text { for } \quad 0<E_{\perp}<E_{c} \\
J_{R}^{*}\left(E_{\perp}\right)=J_{R}\left(E_{\perp}\right) \quad \text { for } \quad E_{\perp}>E_{c}
\end{gathered}
$$

where the function $J_{\text {env }}\left(E_{\perp}\right)$ in Eq. (20) is the envelope of the family of $r$-lines in the range $r_{1}<r<r_{2}$. ${ }^{2}$ A simple but accurate approximation of this function is 5

$$
J_{\text {env }}^{2}\left(E_{\perp}\right) \equiv J_{r_{1}}^{2}\left(E_{\perp}\right)-\frac{2 m_{e} e^{2}\left(r_{1}^{2} \Phi_{1}-r_{0}^{2} \Phi_{0}\right)^{2}}{e\left(r_{1}^{2} \Phi_{1}-r_{0}^{2} \Phi_{0}\right)+\left(r_{0}^{2}-r_{1}^{2}\right) E_{\perp}},
$$

with $r_{1}$ and $\Phi_{1}$ given below by Eqs. (24) and (25). The energy $E_{c}$ appearing in Eq. (20) corresponds to the intersection of the envelope $J_{\text {env }}\left(E_{\perp}\right)$ and the $J_{R}\left(E_{\perp}\right)$ line in the $J^{2}-$ $E_{\perp}$ diagram [see panel (b) in Fig. 2] or

$$
J_{\text {env }}\left(E_{c}\right)=J_{R}\left(E_{c}\right) \approx J_{R}(0) .
$$

b. Quasineutral region with potential barriers. If the radius is decreased beyond $r_{0}$, the potential can still be computed with the quasineutrality relation $N_{e} \approx N_{i}$ but the condition $J_{r}\left(E_{\perp}\right)=J_{r}^{*}\left(E_{\perp}\right)$ does not hold in general because of the appearance of potential barriers. For each r, the relation $J_{r}^{*}\left(E_{\perp}\right)=J_{r}\left(E_{\perp}\right)$ applies in Eq. (18) for $E_{\perp}$ above the point where the r-line touches the envelope and $J_{r}^{*}\left(E_{\perp}\right)=$ $J_{\text {env }}\left(E_{\perp}\right)$ otherwise. ${ }^{6}$ This set of equations is only valid up to a radius $r_{1}$ where the electric field diverges, $(d \Phi / d r$ $\rightarrow-\infty)$. The radius $r_{1}$ and the potential $\Phi_{1}$ can then be determined using the quasineutrality relation and the derivative of the quasineutrality relation with respect to $\Phi$ at $r_{1}$ (where $d r / d \Phi$ vanishes). These two equations read

$$
\begin{aligned}
1= & \exp \left(\frac{e \Phi_{1}}{k T_{i}}\right) \int_{0}^{+\infty} \frac{\exp \left(-E_{\perp} / k T_{e}\right) d E_{\perp}}{\pi k T_{e}} \\
& \times\left[2 \arcsin \left(\frac{J_{e n v}\left(E_{\perp}\right)}{J_{r 1}\left(E_{\perp}\right)}\right)-\arcsin \left(\frac{J_{R}^{*}\left(E_{\perp}\right)}{J_{r 1}\left(E_{\perp}\right)}\right)\right],
\end{aligned}
$$

$$
\begin{aligned}
1= & \exp \left(\frac{e \Phi_{1}}{k T_{i}}\right) \int_{0}^{+\infty} \frac{T_{i} \exp \left(-E_{\perp} / k T_{e}\right) d E_{\perp}}{2 \pi T_{e}\left(E_{\perp}+e \Phi_{1}\right)} \\
& \times\left[\frac{2 J_{e n v}\left(E_{\perp}\right)}{\sqrt{J_{r_{1}}^{2}\left(E_{\perp}\right)-J_{e n v}^{2}\left(E_{\perp}\right)}}-\frac{J_{R}^{*}\left(E_{\perp}\right)}{\sqrt{J_{r_{1}}^{2}\left(E_{\perp}\right)-J_{R}^{* 2}\left(E_{\perp}\right)}}\right],
\end{aligned}
$$

where we used $J_{r 1}^{*}\left(E_{\perp}\right) \approx J_{\text {env }}\left(E_{\perp}\right)$. Equations (19), (23), (24), and (25) give $e \Phi_{0} / k T_{i}, e \Phi_{1} / k T_{i}$,

$$
\sigma_{j} \equiv \frac{m_{e} c^{2}}{k T_{i}} \frac{(1+\beta)^{2}-1}{2}\left(\frac{R}{r_{j}}\right)^{2}, \quad j=0,1
$$

as a function of $T_{e} / T_{i}$ and $E_{c} / k T_{e}$.

\section{Transitional layers}

Following Refs. 3 and 5, we now introduce two transitional layers:

a. First layer of non-quasineutral plasma with potential barrier. Due to the singularity at $r_{1}$, it is necessary to solve Poisson equation with the right-hand side expanded around point 1. Solving for the structure of this layer shows that the potential itself diverges at a point 2 given by

$$
\begin{aligned}
\sigma_{2} & \equiv \frac{m_{e} c^{2}}{k T_{i}} \frac{(1+\beta)^{2}-1}{2}\left(\frac{R}{r_{2}}\right)^{2} \\
& \approx \sigma_{1}\left[1+6.9\left(\frac{2 \sigma_{1}^{2}}{\lambda \mu}\right)^{1 / 5}\left(\frac{k T_{i}}{m_{e} c^{2}} \frac{2}{(1+\beta)^{2}-1}\right)^{2 / 5}\left(\frac{\lambda_{D i}}{R}\right)^{4 / 5}\right],
\end{aligned}
$$

where $\mu$ and $\lambda$ are functions of $E_{c} / k T_{e}$ and $T_{e} / T_{i}$,

$$
\begin{aligned}
\mu \equiv & \int_{0}^{\infty} \frac{\exp \left(-E_{\perp} / k T_{e}\right) d E_{\perp}}{\pi k T_{e}} \times\left[2 \sqrt{\frac{J_{\text {env }}^{2}\left(E_{\perp}\right)}{J_{r_{1}}^{2}\left(E_{\perp}\right)-J_{\text {env }}^{2}\left(E_{\perp}\right)}}\right. \\
& \left.-\sqrt{\frac{J_{R}^{* 2}\left(E_{\perp}\right)}{J_{r_{1}}^{2}\left(E_{\perp}\right)-J_{R}^{* 2}\left(E_{\perp}\right)}}\right], \\
\lambda \equiv & -\exp \left(-\frac{e \Phi_{1}}{k T_{i}}\right)+\int_{0}^{\infty} \frac{k T_{i}^{2} \exp \left(-E_{\perp} / k T_{e}\right) d E_{\perp}}{4 \pi T_{e}\left(E_{\perp}+e \Phi_{1}\right)^{2}} \\
& \times\left[2 J_{\text {env }}\left(E_{\perp}\right) \frac{3 J_{r 1}^{2}\left(E_{\perp}\right)-2 J_{\text {env }}^{2}\left(E_{\perp}\right)}{\left[J_{r 1}^{2}\left(E_{\perp}\right)-J_{e n v}^{2}\left(E_{\perp}\right)\right]^{3 / 2}}\right. \\
& \left.-J_{R}^{*}\left(E_{\perp}\right) \frac{3 J_{r 1}^{2}\left(E_{\perp}\right)-2 J_{R}^{* 2}\left(E_{\perp}\right)}{\left[J_{r 1}^{2}\left(E_{\perp}\right)-J_{R}^{* 2}\left(E_{\perp}\right)\right]^{3 / 2}}\right]
\end{aligned}
$$

b. Second layer of non-quasineutral plasma with potential barrier. The blow up of the potential at point 2 requires a second layer to smoothly match the outer and inner solutions. An analysis of Poisson equation but retaining the full expression for $N_{e}$ and $N_{i}$ reveals that $\Phi \sim\left(r_{2}-r\right)^{4 / 3}$ as $r \rightarrow r_{2}$. 
We remark that all the integrals involving $J_{R}^{*}\left(E_{\perp}\right)$ must be split in energy ranges according to Eqs. (20) and (21). Note also that, even though the structure of the outer solution is similar to the one found in Ref. 6 , the $J_{R}\left(E_{\perp}\right)$ term has relativistic effects. This feature is the origin of the slightly different definitions of $\sigma_{0}, \sigma_{1}$, and $\sigma_{2}$ with respect to the nonrelativistic case.

\section{Sheath structure}

The inner solution extends from the radius $r_{2}$ to $R$ and requires retaining relativistic effects. In this region we have (i) $e \Phi / k T_{i} \gg 1$ and the ion density can be neglected, (ii) the approximation $J_{r}^{*}\left(E_{\perp}\right) \approx J_{e n v}\left(E_{\perp}\right)$ is valid, and (iii) $J_{e n v}\left(E_{\perp}\right) \sim J_{R}(0) \ll J_{r}\left(E_{\perp}\right) \approx J_{r}(0)$ (the arcsin functions in the electron density are approximated by their arguments). The Poisson equation reads

$$
\begin{aligned}
\frac{\lambda_{D i}^{2}}{r} \frac{d}{d r}\left(r \frac{d}{d r} \frac{e \Phi}{k T_{i}}\right) \approx & \frac{\kappa R}{\pi r}\left(1+\frac{e \Phi}{m_{e} c^{2}}\right)^{3 / 2} \\
& \times \sqrt{\frac{(1+\beta)^{2}-1}{\left(1+\frac{e \Phi}{m_{e} c^{2}}\right)^{2}-1}},
\end{aligned}
$$

with $\kappa$ a function of $T_{e} / T_{i}$ and $E_{c} / k T_{e}$

$$
\kappa \equiv \int_{0}^{\infty} \frac{d E_{\perp}}{k T_{e}} \exp \left(-\frac{E_{\perp}}{k T_{e}}\right)\left[2 \frac{J_{e n v}\left(E_{\perp}\right)}{J_{R}(0)}-\frac{J_{R}^{*}\left(E_{\perp}\right)}{J_{R}(0)}\right] .
$$

Introducing the new variable $u$ and the parameter $\alpha$

$$
\begin{gathered}
u \equiv \ln \frac{r_{2}}{r}, \\
\alpha \equiv \frac{R}{\lambda_{D i}} \frac{r_{2}}{\lambda_{D i}} \frac{\kappa}{\pi} \sqrt{(1+\beta)^{2}-1} .
\end{gathered}
$$

Eq. (30) becomes

$$
\frac{d^{2}}{d u^{2}} \frac{e \Phi}{k T_{i}}=\alpha e^{-u}\left[\frac{\left(1+\frac{e \Phi}{m_{e} c^{2}}\right)^{3}}{\left(1+\frac{e \Phi}{m_{e} c^{2}}\right)^{2}-1}\right]^{1 / 2}
$$

\section{B. Results}

\section{Maximum radius for $O M L$ conditions}

The maximum radius of the probe that still collects OML current is very important for bare-tether technological applications. Such a radius is obtained by setting $E_{c}=0$ in the asymptotic analysis and integrating Eq. (34) as a boundary value problem with $R / \lambda_{D i}$ the shooting variable. This dimensionless radius is varied until a numerical integration of Eq. (34) with initial condition $\Phi=0$ and $d \Phi / d u=0$ at $u=0$ (matching with the top of the second thin layer where the potential behaves as $\Phi \sim u^{4 / 3}$ as $u \rightarrow 0$ ) gives $\Phi=\Phi_{p}$ at $u=\ln \left(r_{2} / R\right)$. The solution of this problem gives $R_{\max } / \lambda_{D i}$ as a function of $k T_{i} / m_{e} c^{2}, T_{e} / T_{i}$, and $e \Phi_{p} / k T_{i}$. Panels (a) and (b) in Fig. 3 display the normalized maximum radius $R_{\max } / \lambda_{D i}$ versus $e \Phi_{p} / k T_{i}$ for different temperatures ratios and $k T_{i} / m_{e} c^{2}$ equal to $10^{-4}$ and $10^{-5}$, respectively. The comparison of these panels with Fig. 6 in Ref. 5 shows that the relativistic effects make the ratio $R_{\max } / \lambda_{D i}$ to present a maximum in addition to the minimum also found in Ref. 5.

\section{Sheath radius for $\boldsymbol{R} \ll \boldsymbol{R}_{\max }$}

In the case of a mission in Jupiter, we have $R \ll R_{\max }$ and we can set $E_{c}=0$. From a numerical point of view, the calculation is similar to the case of the maximum radius. However, now the ratio $R / \lambda_{D i}$ is given and $\sigma_{0}$ is taken as shooting variable to solve the boundary value problem. The result is the sheath radius, say $r_{1} / \lambda_{D i}$, as a function of $R / \lambda_{D i}$, $T_{e} / T_{i}, k T_{i} / m_{e} c^{2}$, and $e \Phi_{p} / m_{e} c^{2}$.

Figure 4 shows the ratio $r_{1} / \lambda_{D i}$ versus $e \Phi_{p} / m_{e} c^{2}$ for two different values of $k T_{i} / m_{e} c^{2}$ and parameters $T_{e} / T_{i}=1$ and $R / \lambda_{D i}=0.01$. These results can be compared with nonrelativistic calculations that give the following law for the sheath radius $r_{s}:{ }^{16}$

$$
1.53\left[1-2.56\left(\frac{\lambda_{D i}}{r_{s}}\right)^{4 / 5}\right]\left(\frac{r_{s}}{\lambda_{D i}}\right)^{4 / 3} \ln \left(\frac{r_{s}}{R}\right) \approx \frac{e \Phi_{p}}{k T_{e}} .
$$

The above formula, which is valid for $R \ll R_{\max }$ and high bias, is plotted in Fig. 4 using thin black lines. The results practically overlap the relativistic calculations, indicating a weak impact of the relativistic effect on the sheath radius.
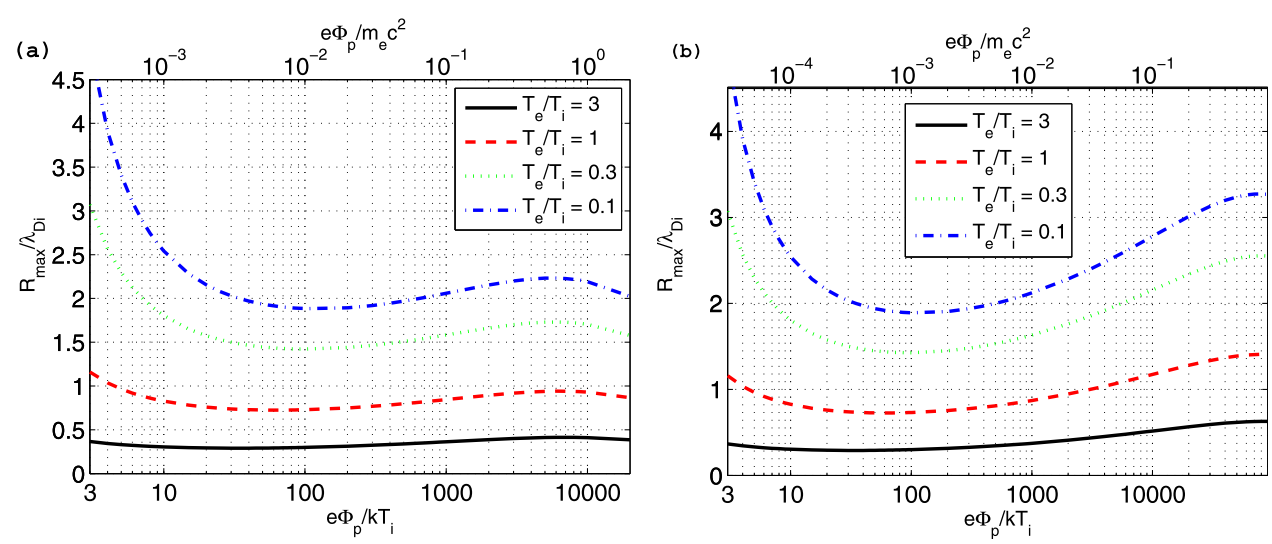

FIG. 3. Asymptotic analysis results: $R_{\max } / \lambda_{D i}$ versus $e \Phi_{p} / k T_{i}$ (bottom horizontal axis) or $e \Phi_{p} / m_{e} c^{2}$ (top horizontal axis) for several values of temperature ratio $T_{e} / T_{i}$. Panels (a) and (b) correspond to $k T_{i} / m_{e} c^{2}$ equal to $10^{-4}$ and $10^{-5}$, respectively. 


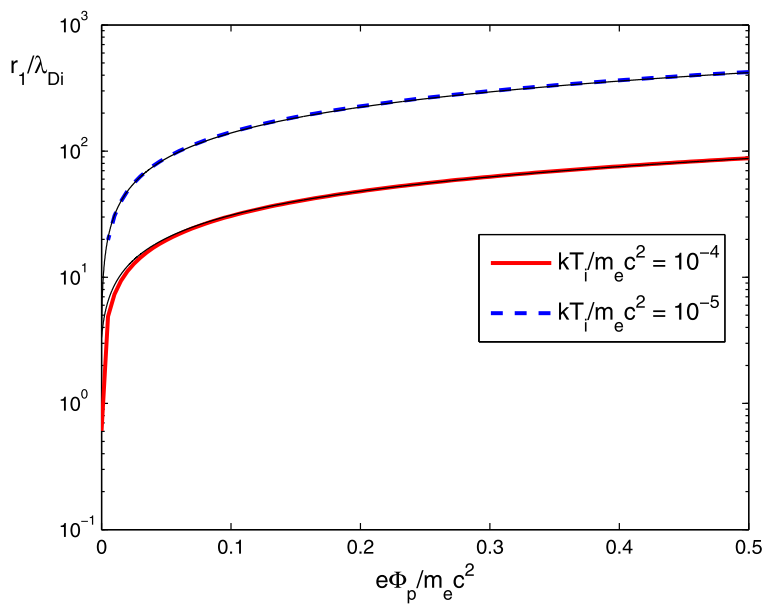

FIG. 4. Asymptotic analysis results: sheath radius $r_{1} / \lambda_{D i}$ versus $e \Phi_{p} / m_{e} c^{2}$ for $k T_{i} / m_{e} c^{2}=10^{-4}, 10^{-5}$. Other parameter values are $T_{e} / T_{i}=1$ and $R / \lambda_{D i}=0.01$. The thin solid lines, which practically overlap with the thick lines, correspond to the nonrelativistic calculations [Eq. (35)].

\section{Current beyond the OML regime}

Even though a bare tether in orbit around Jupiter would find a Debye length and $T_{e} / T_{i}$ ratio varying along the orbit, it would normally operate under OML conditions. However, we give here for completeness the current beyond this regime. The numerical calculations are similar to the case of the maximum radius except that now $E_{c} \neq 0$ and Eq. (23) must be included. For convenience, we choose the energy $E_{c}$ as the shooting variable. This procedure gives the ratio $I / I_{O M L}$ as a function of $R / \lambda_{D e}, e \Phi_{p} / k T_{i}, k T_{i} / m_{e} c^{2}$, and $T_{e} / T_{i}$.

Fig. 5 shows $I / I_{O M L}$ versus $R / \lambda_{D e}$ for $e \Phi_{p} / k T_{i}=4000$, $k T_{i} / m_{e} c^{2}=10^{-4}\left(\beta \equiv e \Phi_{p} / m_{e} c^{2}=0.4\right)$, and different $T_{e} / T_{i}$ ratios. A comparison with Fig. 4 in Ref. 6 reveals a weak impact of the relativistic effects on the ratio $I / I_{O M L}$. However, since $I_{O M L}$ is enhanced by the relativistic effects [see Fig. 1 and Eq. (15)] the current collected by the probe beyond the OML regime is higher as compared with the classical result. Similarly to the nonrelativistic case, the lines in Fig. 5 can approximately be obtained from each other by a horizontal displacement which only depend on the temperature ratio $T_{e} / T_{i}$. This property would allow to simplify the parametric dependence of $I / I_{O M L}$ and find a more simple law

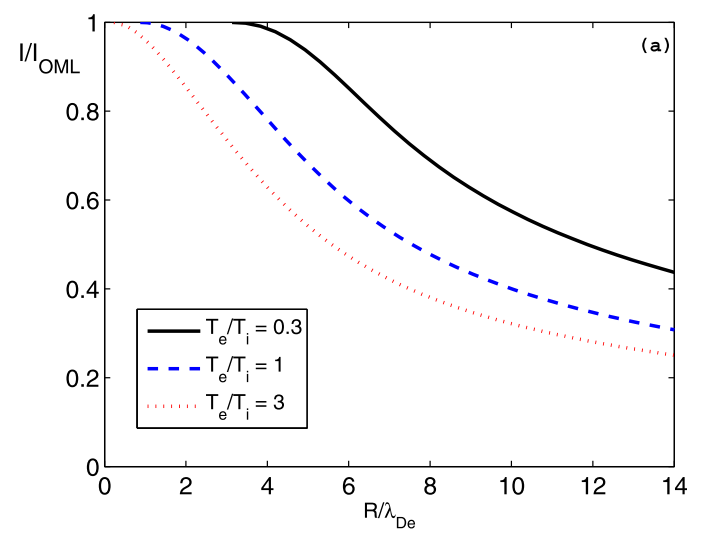

FIG. 5. Asymptotic analysis results: current ratio $I / I_{O M L}$ versus $R / \lambda_{D e}$ for $e \Phi_{p} / k T_{i}=4000, k T_{i} / m_{e} c^{2}=10^{-4}$, and different $T_{e} / T_{i}$ ratios. for design considerations. ${ }^{6}$ On the other hand, when the parameter $e \Phi_{p} / m_{e} c^{2}$ is varied for fixed values of $k T_{i} / m_{e} c^{2}$ and $T_{e} / T_{i}$ (not shown in Fig. 5) a weak effect in $I / I_{O M L}$ is produced.

\section{NUMERICAL VLASOV-POISSON SOLVER}

This section presents numerical solutions of the relativistic Vlasov-Poisson system with an algorithm similar to the one implemented in Refs. 4 and 11. The method truncates the semi-infinite domain $[R, \infty)$ up to a maximum radius $r_{\max }$. The interval $\left[R, r_{\max }\right]$ and the potential $\Phi$ are discretized with $\mathrm{N}$ points according to $r_{i}=R+i\left(r_{\max }-R\right) /(N-1)$ and $\Phi_{i}=\Phi\left(r=r_{i}\right), i=0, \ldots N-1$. The potential $\Phi$ at the mesh points is found by looking with a Newton method for the zero of a vector-function of components $F_{i}(\Phi)=$ $\Phi_{i}-\tilde{\Phi} i(\mathrm{i}=0, \ldots, \mathrm{N}-1)$. Given a potential profile $\Phi$, the electron density is computed with Eq. (10) and then used to find a new potential $\tilde{\Phi}$ by solving Eq. (1) with the boundary conditions $\tilde{\Phi}=\Phi_{p}$ at $r=R$ and $\tilde{\Phi} \sim 1 / r$ at $r_{\max }$. We remark that the Newton algorithm requires the computation of the Jacobian of $\mathbf{F}(\Phi)$ (carried out numerically) and the solution of a linear system of size $N$. Hereafter we fix $k T_{i} / m_{e} c^{2}=$ $10^{-4}$ and $T_{e} / T_{i}=1$.

The number of grid points $N$ was 200 or greater and $r_{\max }$ took values up to $250 \lambda_{D i}$. To validate the Vlasov-Poisson solver by comparing with the asymptotic analysis, we set $e \Phi_{p} / k T_{i}=4000(\beta=0.4)$ and computed the collected current for $R / \lambda_{D i}=1,2.5,5$, and 10 . We found $I / I_{O M L}=0.99$, $0.93,0.70$, and 0.42 , respectively, in very good agreement with the results shown in Fig. 5. Similar to the non-relativistic calculations, ${ }^{11}$ the Vlasov-Poisson solver gives a ratio $I / I_{O M L}$ slightly greater than the asymptotic theory.

As shown in Sec. III [see panel (a) in Fig. 2], a plot of the potential versus $(R / r)^{2}$ readily reveals the current collection regime of the probe: if the potential is above the separatrix given by Eq. (13) (that simplifies to $\Phi / \Phi_{p}=(R / r)^{2}$ in nonrelativistic conditions) the probe collects the OML current. To illustrate this feature, Fig. 6 displays the potential profile

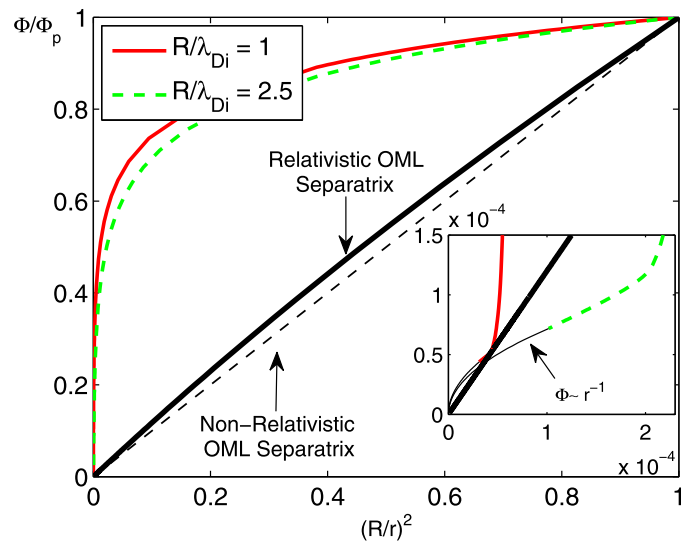

FIG. 6. Vlasov-Poisson solver results: normalized probe potential versus $(R / r)^{2}$ for two $R / \lambda_{D i}$ ratios. Other parameter values are $k T_{i} / m_{e} c^{2}=10^{-4}$, $T_{e} / T_{i}=1$, and $e \Phi_{p} / m_{e} c^{2}=0.4$. The relativistic (see Eq. (13)) and nonrelativistic OML separatrices are also displayed. The thin solid lines in the inset, which is a zoom close to the origin, correspond to potentials proportional to $r^{-1}$. 


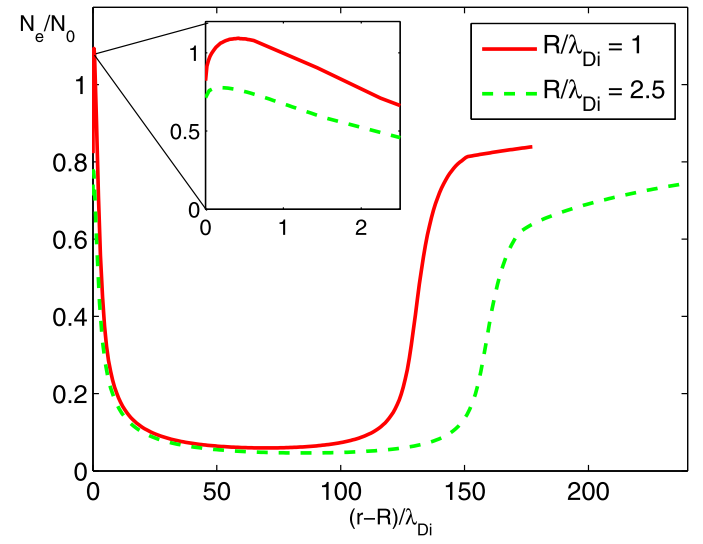

FIG. 7. Vlasov-Poisson solver results: normalized electron density versus $(r-R) / \lambda_{D i}$ for two different probe radius. Other parameter values are at $k T_{i} / m_{e} c^{2}=10^{-4}, T_{e} / T_{i}=1$ and $e \Phi_{p} / m_{e} c^{2}=0.4$.

computed with the Vlasov-Poisson solver for $e \Phi_{p} / m_{e} c^{2}$ $=0.4$. A look at the behavior of the potential far away from the probe (see inset in Fig. 6) reveals that the potential with $R / \lambda_{D i}=1$ is practically tangent to the separatrix whereas the potential for $R / \lambda_{D i}=2.5$ cuts it. This intersection explains the drop of the ratio $I / I_{O M L}=0.93$ below one for $R / \lambda_{D i}=2.5$. On the other hand, the soft transition between the numerical solution and the potential $\Phi \sim r^{-1}$ (see dashed black lines) indicates the goodness of the value $r_{\max }$ taken to carry out the calculations; the value was large enough to impose the boundary condition $\Phi \sim r^{-1}$ when we solved the Poisson equation. The inset in Fig. 6 highlights the importance of large computation domains in this type of calculations.

The electron density profiles that correspond to the potentials of Fig. 6 are shown in Fig. 7. A maximum in the electron density, already detected numerically ${ }^{11,17}$ and explained theoretically ${ }^{16}$ for non-relativistic conditions, can be clearly seen for the cases $R / \lambda_{D i}=1$ and $R / \lambda_{D i}=2.5$ (see inset in Fig. 7). We also point out that the electron density reaches values above $N_{0}$ for the case $R / \lambda_{D i}=1$. This result, that would be impossible within the non-relativistic framework, ${ }^{15}$ is in agreement with the discussion made in Sec. II.

\section{v. CONCLUSIONS}

The impact of the relativistic effects on the current collection by a cylindrical Langmuir probe has been analyzed. This relativistic correction, which is typically very small in laboratory conditions and also for space mission with bare electrodynamic tethers around the Earth, can be important for a recently proposed mission to Jupiter $^{12}$ due to the higher ambient magnetic field, relative plasma-tether velocity, and required tether length arising from constraints on radiation at the Jovian Belts. For typical tether, orbit, and ambient plasma parameters, the dimensionless number that measures the importance of the relativistic effects, $e \Phi_{p} / m_{e} c^{2}$, could reach value of order $0.4-0.5$.

Simple OML-regime calculations showed an enhancement of the collected current when relativistic effects are included [see Eq. (15) and Fig. 1]; i.e., around 35\% for $\beta \sim 0.5$. Equation (10) [also Eq. (16)] shows that, unlike the classical theory, ${ }^{15}$ the electron density could reach values above the unperturbed density. On the other hand, an extension to relativistic conditions of the asymptotic analysis for high bias carried out in Refs. 5 and 6 yielded the maximum radius $R_{\max }$ of a round tether for the OML regime to hold, the sheath radius at low $R / R_{\max }$, and the current for $R>R_{\max }$ (or $w>4 R_{\max }$ for a thin tape of width $w$, Ref. 5). A comparison of Fig. 6 in Ref. 5 and Figure 3 reveals a trending shift at moderate $\beta$ values of the ratio $R_{\max } / \lambda_{D i}$ versus $e \Phi_{p} / k T_{i}$. The value $R_{\max } / \lambda_{D i}$ for $\beta \sim 0.4-0.5$ is not significantly changed and, since the Debye length in Jupiter plasmasphere would typically be $1 \mathrm{~m}$, the tether would operate well within the OML regime. Concerning the sheath radius and the ratio $I / I_{O M L}$, Fig. 4 and a comparison of Fig. 5 with Fig. 4 in Ref. 6, show a weak impact of the relativistic effects. We point out, however, that the collected current beyond the OML regime would be enhanced due to the previously mentioned dependence of $I_{O M L}$ with $\beta$.

The asymptotic theory has been complemented with some numerical results using a Vlasov-Poisson solver. This tool allowed us to compute density and potential profiles and illustrate some differences between the classical and relativistic calculations, in particular the previously mentioned density values above the unperturbed plasma density (see Fig. 7). Figure 6, which shows $\Phi / \Phi_{p}$ versus $R^{2} / r^{2}$ and the relativistic OML separatrix given by Eq. (13), reveals whether or not the probe operates under OML conditions. The inset in this figure also stressed the importance of using large computational domains to obtain correct results.

Possible electron trapping in energy troughs (not considered in our work) as discussed in Ref. 2 involved collisions; collisional effects, which in a lab may affect collection due to the slow $\Phi \approx 1 / r$ decay for a cylindrical probe, are typically negligible for tethers in space. A. V. Gurevich first showed, however, how adiabatic trapping may occur as troughs develop in time. ${ }^{18}$ In the case of a tether, trapped electrons can escape through its ends, or absorbed by the tether, as they move parallel to it and find a radial potential structure lengthwise dependent. Trapping of electrons can only then exist if driven, which the tether-to-plasma relative motion can actually do, as recently pointed out. ${ }^{10,19}$ Orbital velocity is typically highly subsonic for electrons, but supersonic for ions at Earth orbits well below $1000 \mathrm{~km}$. As shown in Ref. 15, $N_{e}<N_{0}$ holds for non-relativistic conditions, whereas the ion ram motion will result in $N_{i}>N_{0}$ over some large front region, breaking quasineutrality in the presheath. It is not yet clear whether this may affect collection. In the relativistic case, however, $N_{e}$ can also exceed $N_{0}$, making driven trapping less of a problem.

\section{ACKNOWLEDGMENTS}

This work was carried out under a grant from the European Commission, FP-7 Space Project No. 262972.

${ }^{1}$ H. M. Mott-Smith and I. Langmuir, "The theory of collectors in gaseous discharges," Phys. Rev. 28, 727-763 (1926).

${ }^{2}$ I. B. Bernstein and I. N. Rabinowitz, "Theory of electrostatic probes in a low-density plasma," Phys. Fluids 2, 112-121 (1959).

${ }^{3}$ S. H. Lam, "Unified theory for the Langmuir probe in a collisionless plasma," Phys. Fluids 8, 73-87 (1965). 
${ }^{4}$ J. G. Laframboise, "Theory of spherical and cylindrical Langmuir probes in a collisionless, Maxwellian plasma at rest," Ph.D. dissertation (University of Toronto, Canada, 1966).

${ }^{5}$ J. R. Sanmartín and R. D. Estes, "The orbital-motion-limited regime of cylindrical Langmuir probes," Phys. Plasmas 6, 395-405 (1999).

${ }^{6}$ R. D. Estes and J. R. Sanmartín, "Cylindrical Langmuir probes beyond the orbital-motion-limited regime," Phys. Plasmas 7, 4320-4325 (2000).

${ }^{7}$ J. G. Laframboise and J. Rubinstein, "Theory of a cylindrical probe in a collisionless magnetoplasma," Phys. Fluids 19, 1900-1908 (1976).

${ }^{8}$ G. V. Khazanov, N. H. Stone, E. N. Krivorutsky, and M. W. Liemohn, "Current-produced magnetic field effects on current collection," J. Geophys. Res. 105, 15835-15842, doi:10.1029/2000JA000039 (2000).

${ }^{9}$ J. R. Sanmartín and R. D. Estes, "Magnetic self-field effects on current collection by an ionospheric bare tether," J. Geophys. Res. 107, 1335, doi:10.1029/2002JA009344 (2002).

${ }^{10}$ T. Onishi, M. Martinez-Sánchez, D. L. Cooke, and J. R. Sanmartín, "PIC computation of electron current collection to a moving bare tether in the mesothermal condition," in 27th International Electric Propulsion Conference, Pasadena, CA, Paper IEPC-01-245, 2001.

${ }^{11}$ E. Choiniere, "Theory and experimental evaluation of a consistent steadystate kinetic model for two-dimensional conductive structures in ionospheric plasmas with application to bare electrodynamic tethers in space," Ph.D. dissertation (University of Michigan, 2004).
${ }^{12}$ M. Charro, J. R. Sanmartin, C. Bombardelli, A. Sanchez-Torres, E. C. Lorenzini, H. B. Garrett, and R. W. Evans, "A proposed two-stage two-tether scientific mission at Jupiter," IEEE Trans. Plasma Sci. 40(2), 274-281 (2012).

${ }^{13}$ D. Hastings and H. Garrett, Spacecraft-Environment Interactions (Cambridge University Press, 1996).

${ }^{14}$ J. R. Sanmartin, M. Charro, E. C. Lorenzini, H. B. Garrett, C. Bombardelli, and C. Bramanti, "Electrodynamic tether at Jupiter-I: Capture operation and constraints," IEEE Trans. Plasma Sci. 36(5), 2450-2458 (2008).

${ }^{15}$ J. G. Laframboise and L. W. Parker, "Probe design for orbit-limited current collection," Phys. Fluids 16, 629-636 (1973).

${ }^{16}$ J. R. Sanmartin, E. Choiniere, B. E. Gilchrist, J.-B. Ferry, and M. Martinez-Sanchez, "Bare-tether sheath and current: Comparison of asymptotic theory and kinetic simulations in stationary plasma," IEEE Trans. Plasma Sci. 36, 2851-2858 (2008).

${ }^{17} \mathrm{~J}$.-B. Ferry, "Electron collection by an electrodynamic bare tether at high potential," Ph.D. dissertation (MIT, Cambridge, 2003).

${ }^{18} \mathrm{~A}$. V. Gurevich, "Distribution of captured particles in a potential well in the absence of collisions," Sov. J. Exp. Theor. Phys. 26, 575 (1968).

${ }^{19}$ J. R. Sanmartín, "Active charging control and tethers," in CNES-Space Technology Course: Prevention of Risks Related to Spacecraft Charging (Toulouse, France, 2002), pp. 515-533. 
Physics of Plasmas is copyrighted by the American Institute of Physics (AIP). Redistribution of journal material is subject to the AIP online journal license and/or AIP copyright. For more information, see http://ojps.aip.org/pop/popcr.jsp 